\title{
Design de Recursos Educacionais Abertos Multiculturais: sobre oportunidades, desafios e possibilidades
}

\author{
Edie Correia Santana ${ }^{12}$, Ismar Frango Silveira1 \\ 1Programa de Pós-Graduação em Engenharia Elétrica e Computação - Universidade \\ Presbiteriana Mackenzie (UPM) - São Paulo - SP - Brasil \\ ${ }^{2}$ Instituto Federal de Educação, Ciência e Tecnologia de Mato Grosso (IFMT) - \\ Campus São Vicente - MT - Brasil \\ ediecs@gmail.com, ismar.silveira@mackenzie.br
}

\begin{abstract}
This work is about the discussions on the topic 3, specifically Multicultural Open Educational Resources. We consider a cultural recontextualization possible and primordial for an Open and Inclusive Education. Therefore, they propose to do this question of systemic and planned form during the process of design of Multicultural Open Educational Resources. We present the expected benefits, strategies to be adopted, the potential challenges as well as the actions to address them.
\end{abstract}

Resumo. A proposta deste trabalho é aprofundar as discussões acerca da linha temática 3, especificamente sobre Recursos Educacionais Abertos (REAs) Multiculturais. Consideramos a recontextualização cultural de REAs desafiadora, porém, possível e primordial para uma Educação Aberta e Inclusiva. Por isso, propomos tratar essa questão de forma sistêmica e planejada durante o processo de design dos REAs Multiculturais. Apresentamos os benefícios esperados, estratégias a serem adotadas, os potenciais desafios, bem como as ações para enfrentá-los.

\section{Introdução}

Os Recursos Educacionais Abertos (REAs) têm atraído cada vez mais atenção e ganhado destaque por ajudar a criar novas situações e possibilidades para a produção e disseminação de conhecimento. O objetivo principal em um REA é oferecer conteúdo educacional por meio dos mais diversos recursos multimídia de forma livre tanto para o acesso quanto para o reuso e adaptação. Isso significa que qualquer pessoa tem permissão legal para baixar e alterar o conteúdo de um REA e, inclusive, compartilhar e distribuir de forma gratuita (ONU, 2018).

O termo REA tem origem nos estudos acerca dos Objetos de Aprendizagem (OA), conforme aponta Wiley (2007). Com a crescente evolução e utilização dos OA, Wiley definiu em 1998 o conceito de Open Content e criou a Open Content License/Open Publication License, visando à massificação dos conceitos do movimento FLOSS (Free Libre and Open Source Software), aplicados ao desenvolvimento de 
conteúdos educacionais. Então, o termo REA engloba o conceito de OA enfatizando o conceito "open". Isso significa que os REA foram pensados para serem mais amplos e evoluirem o conceito de OA, com licença de uso aberta, bem como facilidades técnicas para reuso e adaptação dos seus conteúdos para diferentes contexto e modificados por outros usuários (AMIEL, 2011; WILEY, 2007; ZANCANARO, 2015).

A efetivação dos benefícios esperados com os REAs depende, portanto, não só de questões técnicas, mas também de questões relacionadas com o contexto cultural do estudante que utiliza um REA e até do contexto no qual o REA foi desenvolvido. Desse modo, percebe-se que os elementos culturais estão sempre presentes em um REA seja no uso/reuso ou no processo de desenvolvimento.

Portanto, sabendo que é fundamental representar aspectos culturais em um REA para que ele possa ser significativo e útil para estudantes, como produzir REAs de modo que a recontextualização cultural seja possível ?

Entendemos que uma forma de tratar essa questão de pesquisa, bem como o desafio proposto, é buscar representar os aspectos culturais durante o processo de design, e isso significa buscar uma solução por meio de um enfoque sistêmico e não somente em algumas partes do REAs ou fases do processo de desenvolvimento.

Com isso, este artigo busca contribuir com as discussões e aprofundar os debates dentro da linha temática 3, especificamente sobre os recursos educacionais abertos multiculturais, de modo a contribuir na consolidação deste assunto como um desafio da área de Informática na Educação para o período de 2017 a 2027.

Este artigo está organizado da seguinte forma. A Seção 2 apresenta uma discussão acerca dos benefícios esperados com os REAs Multiculturais. A Seção 3 apresenta estratégias e possibilidades de trabalho colaborativo no design de REAs Multiculturais, na Seção 4 é feita a análise sobre os potenciais desafios no design de REAs Multiculturais, na Seção 5 são apresentadas propostas de ações que devem ser tomadas para se enfrentar o desafio em um período de 10 anos e, finalmente, na Seção 5 são apresentadas as conclusões do trabalho.

\section{Os benefícios esperados dos REAs Multiculturais}

Neste trabalho são considerados aspectos culturais as normas de comportamento, conhecimento, valores morais, estilos, mitos, entre outros (D'AMBROSIO, 2005). Esses aspectos são variáveis de acordo com cada contexto cultural, ou seja, em cada cultura, portanto, existe um modo de falar, de pensar, de agir e de se relacionar com outros indivíduos e isso pode influenciar a aprendizagem, a capacidade e a eficiência para aprender um determinado conteúdo, já que contexto cultural se refere a diferentes aspectos, tais como os aspectos sociais, ideológico, político, étnico, as diferentes línguas, valores, normas e as questões relacionadas ao gênero (GASPARINI, 2013). 
Tratar esses aspectos culturais em REAs, além de cumprir uma das premissas originais de um REA, que é eliminar barreiras no acesso ao conhecimento, significa ter efetivamente REAs reutilizáveis, remixáveis e readaptáveis. Outros benefícios também podem ser gerados, mas não se limitando a esses:

1. Melhorias na acessibilidade dos REAS;

2. Inclusão Social;

3. Inclusão Digital;

4. Conteúdos Educacionais Personalizados;

5. Padrões Abertos;

6. Evolução das licenças abertas;

7. REAs mais sustentáveis;

8. Reuso mais efetivo;

9. Usuários mais participativos;

10. Evolução e/ou criação de modelos, metodologias, teorias, tecnologias e ferramentas;

11. REAs Multiculturais mais ricos em termos de recursos multimídia;

12. Novos modelos de negócios baseados no conceito openness;

13. Maior engajamento das partes envolvidas como professores, alunos e designers;

14. Economia de recursos, entre outros.

Certamente, alguns desses benefícios listados podem ser alcançados de forma imediata, enquanto outros levam mais tempo para serem alcançados, mas para que esses benefícios se concretizem, as dificuldades e desafios inerentes a esse tema devem ser enfrentados tanto pela academia quanto pela indústria, em prol de uma educação mais acessível, desde as situações escolares formais até os contextos mais informais ou vulneráveis.

\section{Estratégias e possibilidades de trabalho colaborativo no design de REAs Multiculturais}

A colaboração possui um papel fundamental não somente na construção do conhecimento, mas também em contextos de trabalho. Neste artigo consideramos o design como um trabalho que não aborda apenas a estética ou desenho, mas engloba vários fatores necessários à concepção de um objeto. Portanto, a colaboração no design significa o ato de trabalhar ou agir de forma conjunta para a concepção de algo ou um objeto, no nosso caso REAs Multiculturais (COELHO, 2011; MCDONNELL, 2010).

$\mathrm{Na}$ literatura podemos encontrar trabalhos que apresentam ideias de trabalhos colaborativos no processo de design de REAs nos quais a colaboração exerce papel 
fundamental tanto na representação de aspectos culturais quanto na condução dos trabalhos do design do REA.

Amiel et al. (2011) propõe um modelo chamado de n-Cultura no qual os designers sabem que o recurso poderá ser utilizado em diferentes contextos e, por isso, trazem ao grupo de desenvolvimento pessoas com a necessária diversidade. Segundo os autores, se o REA for desenvolvido para usuários de três países, espera-se que ao menos um representante ou expert de cada país faça parte da equipe, assim cada nacionalidade ou público-alvo estaria representada no design do REA.

Nessa direção, Rodés et al. (2014) apresentam o processo que apoiou a produção de REAs no contexto do projeto LATIn ${ }^{1}$. O processo consiste na formação de grupos, que irão trabalhar na concepção de REAs baseados em livros abertos escritos de forma colaborativa, na escrita do conteúdo de aprendizagem e por fim na liberação do REAs para acesso e download. Os REAs produzidos possuem como público-alvo estudantes de Instituições de Ensino Superior da América Latina. A base de todo esse projeto é o trabalho colaborativo que tem como suporte uma plataforma online para espaço de trocas e interações entre os componentes dos grupos formados. A figura 1 ilustra $o$ processo proposto.

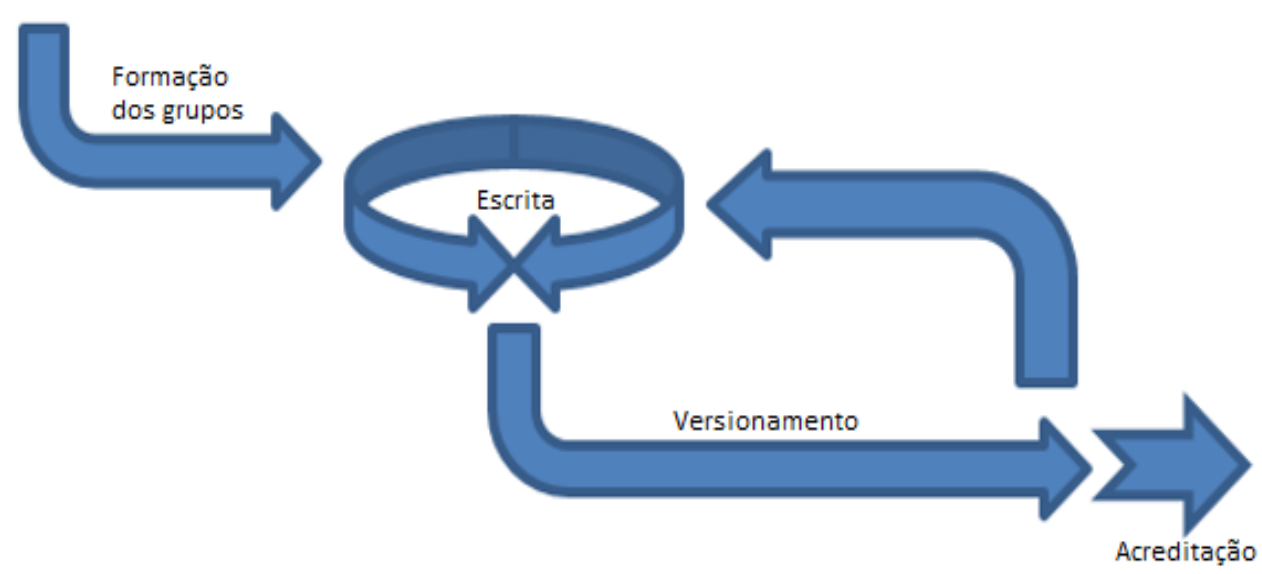

Figura 1: Processo colaborativo do projeto Latin. Adaptada de Rodés et al. (2014).

Tanto o modelo proposto por Amiel et al. (2011) quanto o processo apresentado por Rodés et al. (2014) pressupõem que o REA poderá ser utilizado em diferentes contextos e, por isso, o envolvimento de "representantes" do público-alvo no processo de design é fundamental. Essa vertente de envolvimento das partes é uma iniciativa interessante de exemplo de trabalho colaborativo, embora existam diferentes teorias e modelos de colaboração que podem fornecer outras perspectivas de como se trabalhar em grupos (FUKS et al., 2011).

\footnotetext{
${ }^{1}$ O Projeto LATIn propõe a formação de grupos de professores e pesquisadores para a criação de livros didáticos abertos em diferentes áreas do conhecimento para estudantes de Instituições de Ensino Superior na América Latina (OCHÔA et al., 2011)
} 


\section{Potenciais desafios no design de REAs Multiculturais}

Um importante referencial para esta seção é a declaração feita no $2^{\circ}$ congresso Mundial de REA (UNESCO, 2017) realizado em 2017 na qual se afirma que:

Um REA deve estar disponível em diversas línguas, principalmente nas menos usadas, como línguas indígenas. Também deve ser adaptado ao contexto cultural relacionado, onde é usado para compreensão em contextos locais. Além disso, para que os REAs sejam amplamente utilizados, o compartilhamento e o uso de conhecimento de diferentes fontes precisam ser aceitos pelos interessados em educação. $\mathrm{O}$ apoio e a ação, em particular de governos, instituições educacionais, bem como associações profissionais, inclusive as responsáveis pela harmonização de idiomas, são particularmente importantes para a realização dessas ações (UNESCO, 2017 p. 4).

Com base nesta declaração, mas também em pesquisas sobre a temática (ACEDO; OSUNA, 2016; SANTANA; SILVEIRA, 2017; OCHOA et al., 2011; NWAGWU, 2013; LEINONEN et al., 2010; MORALES-MORGADO et al., 2016; AMIEL, 2013; AMIEL; SOARES, 2016) são considerados potenciais desafios no design de REAs Multiculturais os itens a seguir, não se limitando a esses:

a) A falta de apoio para o desenvolvimento colaborativo de REA multiculturais;

b) A falta de capacitação de educadores e designers para lidar com aspectos culturais como gênero, idiomas, indígenas, entre outros;

c) A pouca participação das partes envolvidas no design de REAs multiculturais como professores, designers, alunos, entre outros interessados;

d) Pouco uso ou criação de tecnologias que ajudam a superar as barreiras linguísticas;

e) Falta de políticas de incentivo para produção de REAs multiculturais;

f) O baixo uso de padrões e licenças abertas no processo de design;

g) A falta de atenção aos princípios do "Openess;

h) Pouca aplicação dos conceitos relacionados a uma Educação Inclusiva;

i) A falta de investimentos públicos em infra-estrutura como de largura de banda para proporcionar maior para comunidades rurais e/ou remotas;

j) A falta de formatos acessíveis para que REA multiculturais possam ser utilizados por todos, incluindo pessoas com deficiências, usando as diretrizes internacionais existentes para acessibilidade.

\section{Ações que devem ser tomadas para se enfrentar o desafio em um período de 10 anos}

As ações a serem tomadas para enfrentar esse desafio incluem: 
- Conscientizar educadores sobre o tema e as vantagens e benefícios de ter REAs que podem ser adaptados para diferentes contextos culturais;

- Capacitar professores e alunos para acessar, reusar, adaptar e compartilhar REAs;

- Fomentar a criação de grupos colaborativos para a agir tanto na produção de REAs Multiculturais quanto na divulgação das vantagens e benefícios desses recursos;

- Criar modelos de negócios que tornem a produção de REAs Multiculturais sustentável;

- Criar workshops sobre o tema em eventos nacionais e internacionais;

- Fortalecer e formar grupos de pesquisa na área;

- Organizar repositórios e construir plataformas que possam apoiar o desenvolvimento de REA multiculturais; e

- Sensibilizar agentes públicos sobre o tema e as vantagens e benefícios de uma educação mais aberta e inclusiva.

\section{Conclusões}

Os REAs multiculturais podem oferecer oportunidades para melhorar a aprendizagem, a qualidade de ensino e fortalecer a democratização à educação, disponibilizando materiais de aprendizagem para alunos e educadores e, ao mesmo tempo, oferecendo opções educacionais acessíveis. Para tanto, é fundamental tratar os desafios para a representação de aspectos culturais durante o design de REAs multiculturais, pois isso pode fornecer uma base sólida para apoiar as mais diversas questões culturais da atualidade.

É neste sentido que este trabalho busca contribuir com $7^{\circ}$ Workshop de Desafios da Computação aplicada à Educação, promovendo reflexões e discussões em torno da complexa tarefa de ampliar o acesso à uma Educação de qualidade.

\section{Referências}

Acedo, S. O. and Osuna, S. M. T. (2016). "ECO European project: inclusive education through accessible MOOCs". In: Proceedings of the Fourth International Conference on Technological Ecosystems for Enhancing Multiculturality (TEEM '16), Francisco José García-Peñalvo (Ed.). ACM, New York, NY, USA, 881-886.

Amiel, T., Orey, M. and WEST, R.. (2011). "Recursos Educacionais Abertos: modelos para localização e adaptação”. ETD - Revista Educação Temática Digital, Campinas - SP, v.12, 2011. p.112-125.

Amiel, T. (2013) "Identifying barriers to the remix of translated open educational resources". The International Review of Research in Open and Distributed Learning, [S.1.], v. 14, n. 1, p. 126-144, jan. 2013. ISSN 1492-3831. 
Amiel, T., Soares, T. C. (2016) "Identifying Tensions in the Use of Open Licenses in OER Repositories". The International Review of Research in Open and Distributed Learning, [S.1.], v. 17, n. 3, may. 2016. ISSN 1492-3831.

Coelho, L. Conceitos-chave em design. Rio de Janeiro: Ed. PUC-Rio, 2011.

Chung, S. H. and Khor, E. T. (2015) "Collaborative OER course development: remix and reuse approach". In Companion to the Proceedings of the 11th International Symposium on Open Collaboration (OpenSym '15). ACM, New York, NY, USA, Article 6, 4 pages. 2015.

D‘ambrosio, U. (2005) "Society, Culture, Mathematics and its Teaching”. Educação e Pesquisa. Revista da Faculdade de Educação da USP, 31 (1), 100 -120.

Fatayer, M. (2014) "Unleash the Cognitive Surplus inside classrooms through OER". In: The National OER Symposium, Tasmania - Australia, 2014.

Fuks, H., Raposo, A. B., Gerosa, M.A., Pimentel, M., Filippo, D. and Lucena, C.J.P. (2011) "Teorias e modelos de colaboração". In: Pimentel, M. e Fuks, H. (Org.). Sistemas colaborativos. Rio de Janeiro: Elsevier-Campus-SBC.

Gasparini, I. (2013) "Aspectos Culturais no Modelo do Usuário em Sistemas Adaptativos Educacionais: Fundamentos, Proposta e Experimentação". Tese (Doutorado em Computação). Instituto de Informática - UFRGS, 2013.

Leinonen T., Purma J., Põldoja H. and Toikkanen T. (2010) "Information architecture and design solutions scaffolding authoring of open educational resources". IEEE transactions on learning technologies. 116-128.

Mcdonnell, J. (2010) "Codesign: International Journal of CoCreation in Design and the Arts”, Londres, v. 1, n. 1, p.1-4, set. 2010.

Morales-morgado, E. M., Ortuño, R. A. C, and Bonilla, A. A. P (2016) "Multimedia resources to develop information competence: A methodological design for the comparative study of undergraduate and post-graduate students in two public universities in Chile and Spain". Computers in Education (SIIE), 2016 International Symposium on. IEEE.

Nwagwu, W. E. (2013) "Open access initiatives in Africa-structure, incentives and disincentives." The Journal of Academic Librarianship 39.1 (2013): 3-10.

Ochôa, X., Silveira, I. F. and Silva, A (2011) "Collaborative open textbooks for Latin America - The LATIn Project". International Conference on Information Society (iSociety 2011), London, 2011, pp. 398-403.

ONU, Brasil. Recursos educacionais precisam ter licença aberta. Acessado em Março de 2018. [Online]. Disponível em: https://nacoesunidas.org/artigo-recursoseducacionais-precisam-ter-licenca-aberta/.

Rodés, V., Mustaro, P. N., Silveira, I. F., Omar, N. and Ochôa, X. (2014) "Instructional Design Models to support Collaborative Open Books for Open Education”. In Proceedings of the XV International Conference on Human Computer Interaction (Interacción '14). ACM, New York, 7 pages, 2014. 
Santana, E. C. and Silveira, I. F. (2017) "Uma Revisão Sistemática Aplicada ao estudo de Recursos Educacionais Abertos Multiculturais”. In: Anais do Simpósio Brasileiro de Informática na Educação 2017 - SBIE 2017, pp. 153-162.

Unesco. (2017) “Open Educational Resources Open Content For Higher Education Final Forum Report". Disponível em: http://www.oercongress.org/. Acesso em: 01 abr. 2018.

Wiley, D. (2007) "Openess, localization, and the future of learning objects". Disponível em: < http://opencontent.org/presentations/bcnet07/ >. Acesso em: 30 mar. 2018.

Zancanaro, A. (2015)" Produção de Recursos Educacionais Abertos com foco na disseminação do conhecimento: uma proposta de framework". Tese de Doutorado. Programa de Pós-Graduação em Engenharia e Gestão do Conhecimento. Universidade Federal de Santa Catarina. Florianópolis: SC. p. 383. 2015. 